\title{
La maternità in dialisi peritoneale (DP) è possibile
}

\author{
Gianna d'Adamo
}

Nefrologo, Roma

Pregnancy in Peritoneal dialysis (PD) is Possible

Abstract. Both in hemodialysis and peritoneal dialysis (PD) the pregnancies are rare and the risk of adverse events for mother or fetus is increased. Based on recent retrospective series and reviews of cases in PD, the outcomes have improved over time. However, the lack of complete data suggests the need to systematically collect prospective data in order to explore the rates of pregnancies, complications, and outcomes as well as to develop specific recommendations about the dialysis prescriptions and the dialysis adequacy targets for pregnant women.

Key words: Peritoneal dialysis, Pregnancy

Conflict of interest: None.

Financial support: None.

Accettato: 16 Settembre 2014

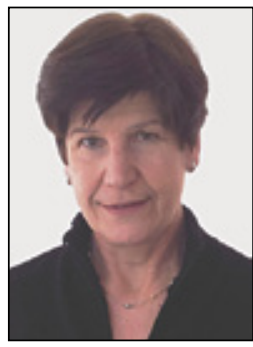

Gianna d'Adamo
In corso di trattamento dialitico le gravidanze sono rare e il rischio che si verifichino eventi avversi sia per la madre che per il feto è aumentato.

Uno studio che ha analizzato i dati del Registro ANZDATA dal 1966 al 2008 ha però riscontrato l'incremento del tasso di gravidanze nel tempo, con percentuale stabile $(79 \%)$ di nascite di un neonato vivo, anche se frequentemente pretermine $(53.4 \%)$ e di basso peso. La probabilità di una gravidanza sarebe però minore In DP rispetto all'emodialisi (1).

Il recente studio retrospettivo italiano condotto dal gruppo di studio "Rene e Gravidanza" della Società Italiana di Nefrologia, con la collaborazione del Registro Italiano Dialisi e Trapianto e dell'Associazione Nazionale Emodializzati, raccoglie i dati relativi alle gravidanze delle donne in dialisi che si sono concluse con la nascita di un neonato vivo nel periodo 2000-2012 e stima che le donne in dialisi abbiano una probabilità di avere un figlio vivo 100 volte inferiore rispetto alle donne della popolazione generale di età compresa tra 20 e 45 anni (2).

Da 20 donne in emodialisi e 3 in dialisi peritoneale sono nati 24 bambini, tra cui 2 gemelli. Il parto è stato quasi sempre prematuro - in un terzo dei casi è avvenuto prima della $34^{\circ}$ settimana - e in 21 casi è stato espletato con taglio cesareo. Un terzo dei bambini sono nati "piccoli per l'età gestazionale" e 3 neonati pretermine non sono sopravvissuti nonostante la terapia intensiva. Circa metà delle madri erano anuriche e alcune erano in dialisi da molti anni. Durante la gravidanza Il trattamento dialitico è stato intensificato in tutti i casi tranne una madre entrata in DP all'inizio della gravidanza (2).

Nella maggior parte dei casi dell'esperienza italiana, come negli altri riportati in letteratura, le madri erano trattate con emodialisi, più raramente con DP, ma non ci sono dati che indichino la superiorità di una tecnica dialitica rispetto all'altra (3). Alcune caratteristiche proprie della DP - stabilità metabolica ed emodinamica, continuità dell'ultrafiltrazione, assenza di episodi ipotensivi e di anticoagulanti - potrebbero facilitare la gestione della gravidanza (4). Per contro, la distensione dell'utero nel $3^{\circ}$ trimestre può comportare difficoltà di drenaggio e la necessità di ridurre il volume di riempimento (5), rendendo più difficile realizzare l'aumento della depurazione che dovrebbe compensare la generazione di urea da parte del feto e ridurre l'entità dell'uremia materna (6).

Nella recente review di Batarse e coll. che raccoglie 47 gravidanze in DP, di cui 37 con esito favorevole, la maggior parte delle prescrizioni dialitiche nel terzo trimestre comprendono la riduzione del volume di riempimento a 1,5 litri, o anche meno, e l'aumento del numero degli scambi, tanto in CAPD quanto in APD, senza mai lasciare l'addome vuoto (5). In un caso è riportata la prescrizione di un regime tidal per un problema di malfunzionamento del catetere e dolore addominale durante il drenaggio (7).

Non ci sono studi che indichino quanto debba essere aumentata la depurazione. Un orientamento può venire da uno studio sulle caratteristiche cliniche e gli esiti di 28 donne 
trattate con emodialisi che mostrava un rapporto inverso tra urea (BUN) materna e età gestazionale o peso alla nascita. Pur non sapendo se proprio i bassi valori di BUN fossero la causa diretta del miglioramento degli esiti, gli autori suggerivano di mantenere il BUN predialisi $<50 \mathrm{mg} / \mathrm{dL}$ e di perseguire questo obiettivo quanto più precocemente possibile in corso di gravidanza (8). Essendo questo target relativo alla concentrazione di picco del BUN, Batarse e coll. ipotizzano che in DP si debba mirare ad un valore ancora più basso (5). Al di là delle opinioni di alcuni esperti, non ci sono però in letteratura gli elementi necessari a definire $i$ target di adeguatezza dialitica in corso di gravidanza, dato che nella maggior parte dei report la funzione renale residua e gli indici di adeguatezza non sono riferiti.

Per quanto attiene alle complicanze del trattamento dialitico, oltre alle frequenti già citate complicanze meccaniche, sono stati segnalati episodi di peritonite e di emoperitoneo che in alcuni casi hanno comportato la perdita del feto $(9,10)$. Per quanto riguarda in particolare l'emoperitoneo, è stato segnalato che un sanguinamento importante può conseguire ad aborto, distacco di placenta o lesioni della superficie esterna dell'utero gravido verosimilmente causate dal catetere e non descritte nella popolazione generale (10).

Se si realizzasse l'auspicio di una raccolta sistematica dei dati relativi alle gravidanze nell'ambito dei registri di dialisi (2), conosceremmo innanzi tutto la reale frequenza delle gravidanze in corso di dialisi e potremmo poi quantizzare la dose dialitica utile a favorirne l'esito positivo.

\section{Riassunto}

Sia in emodialisi che in dialisi peritoneale le gravidanze sono rare e il rischio di eventi avversi per la madre o per il feto è aumentato. Recenti lavori retrospettivi e reviews di casi in DP mostrano un probabile miglioramento degli esiti nel tempo. Tuttavia l'incompletezza dei dati disponibili rende auspicabile la raccolta sistematica di dati prospettici per conoscere la reale frequenza delle gravidanze, delle complicanze e degli esiti e per elaborare raccomandazioni circa la dose dialitica utile a favorire l'esito positivo della gravidanza.

Parole chiave: Dialisi peritoneale, Gravidanza

Dichiarazione di conflitto di interessi: L'Autore dichiara di non avere conflitto di interessi.

Contributi economici agli Autori: L'Autore dichiara di non avere ricevuto sponsorizzazioni economiche per la preparazione dell'articolo.

Indirizzo dell'Autore:

Dr.ssa Gianna d'Adamo

Nefrologa

Roma

giannadadamo@gmail.com

\section{Bibliografia}

1. Shahir AK, Briggs N, Katsoulis J, et al. An observational outcomes study from 1966-2008, examining pregnancy and neonatal outcomes from dialysed women using data from the ANZDATA Registry. Nephrology (Carlton). 2013; 18: 276-84.

2. Piccoli GB, Cabiddu G, Daidone G, et al. The children of dialysis: live-born babies from on-dialysis mothers in Italy-an epidemiological perspective comparing dialysis, kidney transplantation and the overall population. NDT 2014; 29: 1578-86 Epub 2014 Apr 22.

3. Okundaye I, Abrinko P, Hou S. Registry of pregnancy in dialysis patients. Am J Kidney Dis. 1998; 31: 766-73.

4. Redrow M, Cherem L, Elliott J, et al. Dialysis in the management of pregnant patients with renal insufficiency. Medicine (Baltimore). 1988; 67: 199-208.
5. Batarse RR, Steiger RM, Guest S. Peritoneal dialysis prescription during the third trimester of pregnancy. Perit Dial Int, in Press. Epub 2014 Apr 7.

6. Okundaye I, Hou S. Management of pregnancy in women undergoing continuous ambulatory peritoneal dialysis. Adv Perit Dial. 1996; 12: 151-5.

7. Chang H, Miller MA, Bruns FJ. Tidal peritoneal dialysis during pregnancy improves clearance and abdominal symptoms. Perit Dial Int. 2002; 22: 272-74

8. Asam Asamiya Y, Otsubo S, Matsuda Y, et al. The importance of low blood urea nitrogen levels in pregnant patients undergoing hemodialysis to optimize birth weight and gestation age. Kidney Int. 2009; 75: 1217-22.

9. Gadallah MF, Ahmad B, Karubian F, et al. Pregnancy in patients on chronic ambulatory peritoneal dialysis. Am J Kidney Dis. 1992; 20: 407-10.

10. Lew SQ. Persistent hemoperitoneum in a pregnant patient receiving peritoneal dialysis. Perit Dial Int. 2006; 26: 108-11. 